\title{
Analysis and characterization of the PMD camera for application in mobile robotics
}

\author{
Matthias Wiedemann* Markus Sauer* Frauke Driewer* \\ Klaus Schilling* \\ * University of Wuerzburg, Germany \\ Computer Science VII: Robotics and Telematics \\ (Tel: +49-931-888-6769; e-mail: \\ \{driewer,sauer,schi\}@informatik.uni-wuerzburg.de)
}

\begin{abstract}
Three-dimensional perception of the environment offers significant potential to improve navigation solutions for mobile systems. The Pixel-Mixed-Device Technology (PMD) offers here a small, light-weight camera generating 3-D images, based on time-of-flight measurements. This contribution analyzes the sensor characteristics and application potential for mobile robots. Specific aspects related to parameter optimization and limitations in measurements are discussed on basis of performance tests. In particular, range images in unstructured and changing scenes are of interest. Specific properties of mobile systems are taken into account to generate appropriate range images for navigation and mapping tasks in robotic applications. Related adaptations of integration time and methods to percept consistent distances are derived and characterized by tests.
\end{abstract}

\section{INTRODUCTION}

Safe navigation in poorly known environments is a key research area for mobile systems, offering broad application potential, ranging from assistance systems for cars in the streets (cf. Kiencke and Nielsen [2000]) to emergency operations for search and rescue (Driewer et al. [2005], Schilling and Driewer [2005]), from industrial transport robots (Schilling et al. [1998]) to planetary rovers in space exploration (Schilling [1997]). For this purpose, a threedimensional perception of the environment is a significant contribution for improved obstacle avoidance and path planning tasks, for autonomous reactions as well as for tele-operations.

Classical approaches for 3-D-imaging refer to stereocameras, optical flow approaches and laser range scanners, while so far in industrial applications still mainly simple and reliable 2-D-sensors are in use (Everett [1995]). Therefore, the new principle of time-of-flight range cameras to generate 3-D-images attract much interest (Xu et al. [1998], Prusak et al. [2005], Oggier et al. [2005]). PMD-cameras provide depth images, so that every pixel of the image includes beyond the grayscale information also information about the distance between sensor and observed object. These compact cameras can be operated at high frame rates and do not include any mechanical moving parts, being thus very suitable for mobile systems. Nevertheless, for such innovative sensor principles, further research needs to be invested to characterize performance details and to optimize parameter settings for typical application scenarios.

This contribution first provides an overview about 3Dtime-of-flight range cameras and introduces details for one exemplary PMD camera. In the second section measurements related to variations of the crucial parameters are summarized. A new method for improved 3D perception by adaptation of integration time for dynamic scenes is presented. Procedures for calibration and bad pixel removal are introduced. Finally, these results are transferred to mobile robot applications.

\section{3D DISTANCE MEASUREMENT SENSORS}

Today mainly 3D laser scanners are used for 3D mapping in robotics (Sheh et al. [2006], Nüchter et al. [2006]). There exist systems with nodding 2D laser scanner as well as high accurate 3D laser scanners. The frequency of getting point clouds from this types of sensors differ from 10 seconds to 26 minutes depending on the increment of the tilt angle. In other approaches for mobile robots, two or more $2 \mathrm{D}$ laser scanners mounted horizontally or vertically provide a field-of-view of $360^{\circ}$ (Wulf and Wagner [2003]).

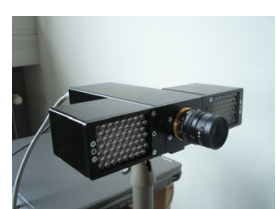

(a)

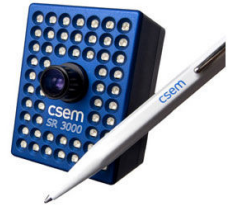

(b)

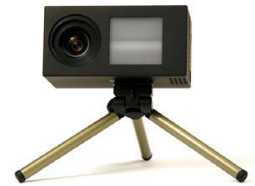

(c)
Fig. 1. Actual range cameras (a) PMD[vision]®19k (http://www.pmdtec.de) (b) CSEM Swissranger 3000 (http://www.swissranger.de) (c) Canesta DP 200 (http://www.canesta.com)

On mobile systems properties like size, weight and power consumption of sensors as well as accuracy, scan-time and range specifications play an important role. Here, the sensor based on the PMD technology promise advantages according to size, weight, and scan-time. Several companies offer range cameras with different resolutions and 
specifications (cf. Fig. 1). In Table 1 the characteristics of several models are shown.

\begin{tabular}{|c|c|c|c|}
\hline model & resolution & outdoor & field of view \\
\hline $\mathrm{PMD}[$ vision] $] 1 \mathrm{k}-\mathrm{S}$ & $64 \times 16$ & SBI $^{1}$ & $45^{\circ} \times 16^{\circ}$ \\
\hline $\mathrm{PMD}[$ vision] $]$ ß $3 \mathrm{k}-\mathrm{S}$ & $64 \times 48$ & SBI $^{1}$ & $40^{\circ} \times 30^{\circ}$ \\
\hline $\mathrm{PMD}[$ vision] $] 19 \mathrm{k}$ & $160 \times 120$ & no & $40^{\circ} \times 30^{\circ}$ \\
\hline PMD [vision] $] \AA A$ & $64 \times 16$ & & $42^{\circ} \times 46^{\circ}$ \\
\hline Swissranger SR 2 & $124 \times 160$ & no & $43^{\circ} \times 46^{\circ}$ \\
\hline Swissranger SR 3000 & $176 \times 144$ & yes $^{2}$ & $47.5^{\circ} \times 39.6^{\circ}$ \\
\hline Canesta EP200 & $64 \times 64$ & & 30,55 or $80^{\circ}(\mathrm{h})$ \\
\hline Canesta DP300 & $64 \times 64$ & $\mathrm{CMR}^{3}$ & 30,81 or $88^{\circ}(\mathrm{h})$ \\
\hline
\end{tabular}

Table 1. Range cameras models available

The camera used in this analysis, a PMD[vision]@19k (Fig. 1(a)) by PMDTechnologies $\mathrm{GmbH}$, Siegen, provides in one measurement $160 \times 120$ pixels with three values per pixel: distance information, amplitude and an 8 bit gray scale value. In each of the pixels the phase shift between emitting and perceiving of a modulated infrared light signal is determined, which is proportional to the distance ( $\mathrm{Xu}$ et al. [1998], Möller et al. [2005]).

The sensor data of this camera can be retrieved by an ethernet IEEE $802.3 \mathrm{u}$ or a firewire IEEE 1394a interface. A software development kit (SDK) for windows and linux systems is available. The camera targets for indoor use only, since technology for dealing with sunlight is not included. In principle, outdoor applications are possible and the appropriate technology is already implemented in other camera models.

\subsection{Related work}

Range cameras In the context of mobile robotics Prusak et al. [2005] present ideas of potential applications. Hybrid systems of a PMD camera and a CCD video camera (Kuhnert and Stommel [2006], Lindner et al. [2007]) or stereo camera (Prasad et al. [2006]) are also investigated. These constructions require calibration of both camera images and can extend PMD data by color information and increased visual resolution.

Calibration Calibration of range cameras is a two-step process, which consists of photogrammetric calibration (like it is done for normal digital cameras) and depth calibration.

Kahlmann et al. [2006] used an extra array of infrared LEDs to improve corner detection of a chessboard to determine the desired camera matrix. Lindner and Kolb [2006] used pixel interpolation and standard tools for calculating the camera matrix.

There are two possibilities for depth calibration: global and pixelwise approximation of the characteristic curve. While only one regression has to be done for the global one, it is necessary to approximate a function for every pixel and each parameter setting. The calibration method has to be chosen depending on accuracy requirements and on time limits for data processing.

\footnotetext{
1 SBI: suppression of background illumination

2 Inherent background light suppression

3 CMR: Common Mode Reset
}

Applications Weingarten et al. [2004] describe obstacle avoidance and path planning with a Swissranger SR2. Hong et al. [2004] also report about obstacle detection for AGVs (Automated Guided Vehicle) in factory environments. Sheh et al. [2006] used a SR2 for map building in an artificial USAR (urban search and rescue) environment. Craighead et al. [2006] tested a camera model from Canesta for application in USAR, in particular regarding lightning conditions and disturbances caused by movements.

Moreover, a PMD camera was used by Ruangpayoongsak [2006] for landmark detection in order to localize a mobile robot. For 3D-SLAM (simultaneous localization and mapping) mainly range measurements from 3D laser scanners have been used. While Weingarten and Siegwart [2005] uses a probabilistic approach (Extended Kalman filter), Nüchter et al. [2005] employs an analytic way (Iterative Closest Point scanmatching).

Besides robotics, many potential applications for automotive, multimedia and other areas have been described (Oggier et al. [2005]). In automotive industry, range cameras are used for crash avoidance, as a parking aid, or as a distance sensor for cruise control. SR2 and a canesta models were also used for a virtual keyboard (Du et al. [2005]) and for an interactive canvas.

\section{CAMERA CHARACTERISTICS}

Two parameters can be adjusted, the modulation frequency and the integration time. Although it is possible to set the modulation frequency, the behavior of the measurement data will be undefined and would require another calibration step. Hence, the analysis of this contribution considers a constant modulation frequency of $20 \mathrm{MHz}$. The other variable parameter is the integration time, which is investigated in the next section.

\subsection{Integration time}

Integration time is the most critical internal parameter of the PMD camera. It describes the time period in which incoming photons are detected for one measurement cycle, in order to derive phase shift and the corresponding distance.

If the integration time is too low, the amplitudes of related pixels decrease and distances for distant objects cannot be measured (Fig. 2(a)). On the other hand, if the integration time is too high, oversaturation is observed and measurements fail, too (Fig. 3). Therefore, a major challenge in obtaining appropriate range images is to find adequate integration time values for particular scenes (Fig. $2(\mathrm{~b})$ and $(\mathrm{c}))$.

While for some camera types the adaptation of integration time is realized by hardware (SR3000: pixelwise integration time $(\mathrm{PWI}))$, for the PMD[vision] $\mathrm{R} 19 \mathrm{k}$ it must be solved by software. May et al. [2006] proposes a proportional controller for the Swissranger SR2, which takes the mean intensity value of the image to adapt integration time to a constant preset value. May et al. [2006] derive a constant relation between intensity and amplitude values. 


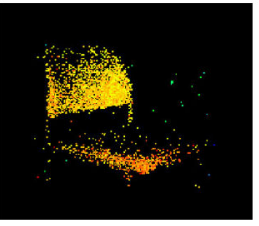

(a) $500 \mu \mathrm{s}$

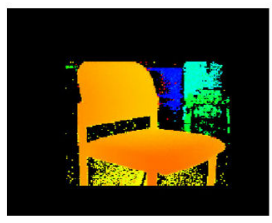

(b) $10.000 \mu \mathrm{s}$

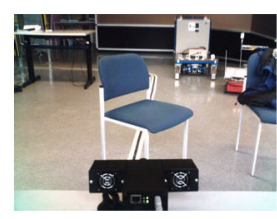

(c) observed scene
Fig. 2. Influence of integration time, distance is coded by color

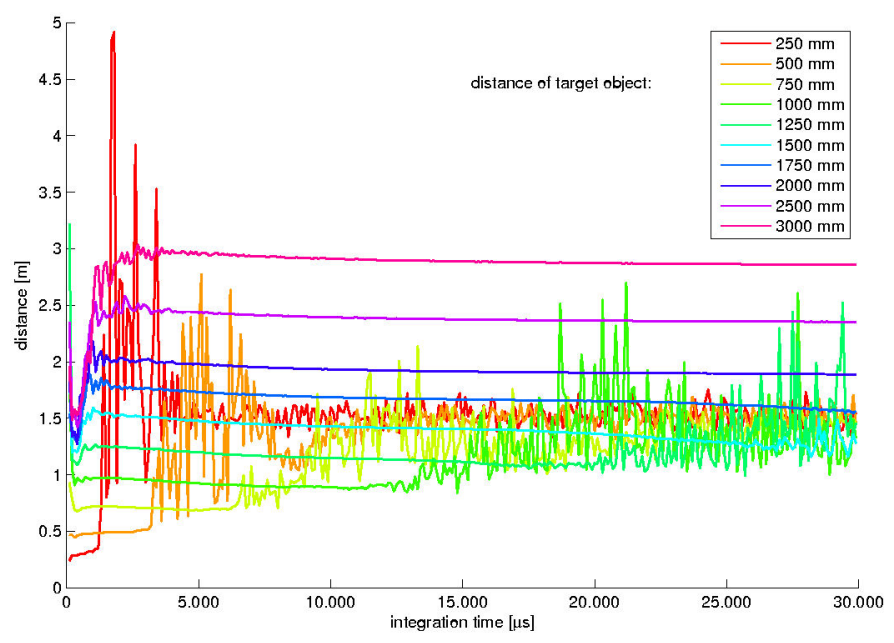

Fig. 3. Measured range for 10 objects in different distances to the PMD camera.

According to measurements with the PMD[vision] (B) $19 \mathrm{k}$ for typical highly dynamic scenes in mobile robotics, May's adaptation scheme cannot be applied. Integration time has to be adapted according to related distances to the objects (cf. Fig. 4). In contrast to the intensity average value, we used the amplitude mean to modify the integration time proportionally. In particular, integration time has to be adapted for near objects $(<1 \mathrm{~m})$ to avoid oversaturation (Fig. 3).

The proposed adaptation method consists of the following steps:

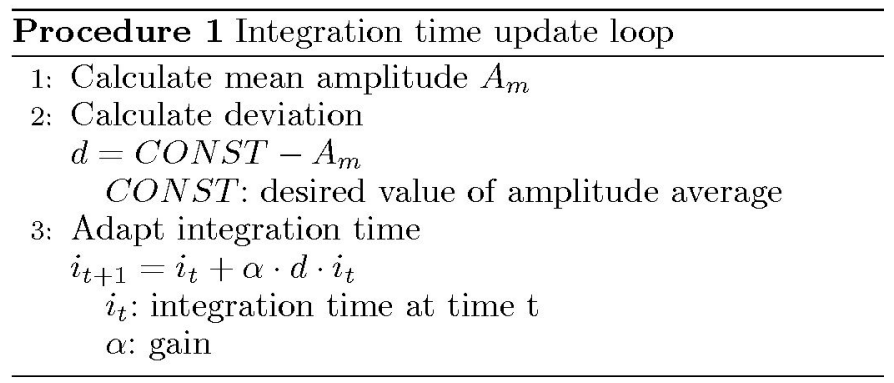

The adaptation of integration time was verified in a testsetup with known distances (cf. Fig. 5). Fig. 6(a) and 6(b) display the measurements for a rotating robot with the predefined velocity of $10^{\circ}$ per second. In both scenarios, moving from near to far objects, as well as from far to near objects, the amplitude converges and correct measurements are obtained. To avoid oversaturation CONST has to be selected according to a desired minimum distance.

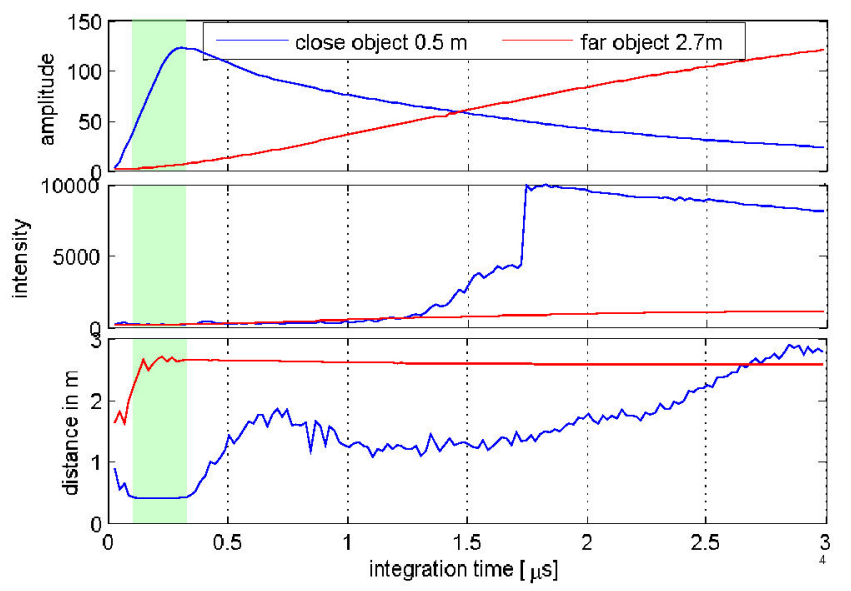

Fig. 4. Measurement plots for the test scene of Fig. 5. Green background indicates correct distance for close object.

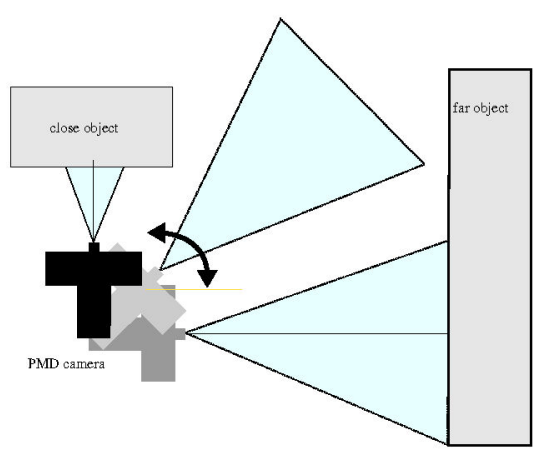

Fig. 5. Test installation for adaptation of integration time. Camera is modeled with central projection scheme.

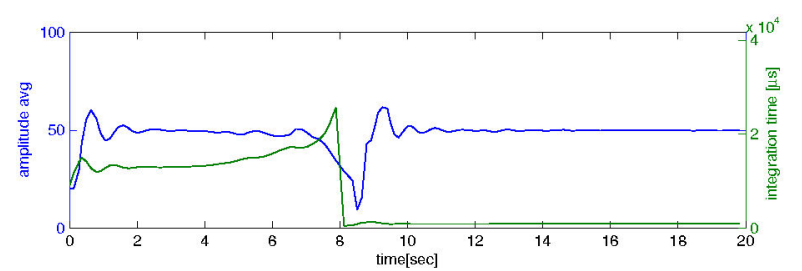

(a)

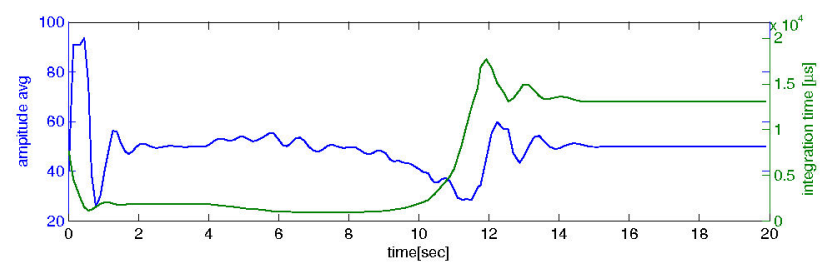

(b)

Fig. 6. Adaptation of integration time (a) from a far object $(2 \mathrm{~m})$ to a near object $(0.5 \mathrm{~m})(\mathrm{b})$ from a near to a far object.

\subsection{Calibration}

For the employed camera, it is possible to set a global calibration shift value by the application programming interface (API) of the SDK. Additionally, a photogrammet- 
ric calibration was realized by a combination of different methods (cf. Section 2.1) with a chessboard.

Important parameters in calibration are horizontal and vertical field of view. With

$$
\alpha=2 \arctan \frac{d}{2 f}
$$

, where $d$ is the width or height of the chip and $f$ is the focal length, a field of view of $29.6^{\circ}$ (horizontal) $/ 22.4^{\circ}$ (vertical) from a focal length of $12.11 \mathrm{~mm}$ was determined. These values are used in the camera matrix for calculating Cartesian coordinates from PMD data.

During the depth calibration, a characteristic curve (cyclic effects) for the measurement deviations could be detected (cf. Fig. 7). We applied a polynomial regression for each pixel to estimate this curve by using a planar white wall as target object.

As mentioned in Section 2.1, calibration for a specific range of integration times is very time consuming, when nominal distances are calibrated by hand. Therefore, precise automatic calibration equipment is desirable.

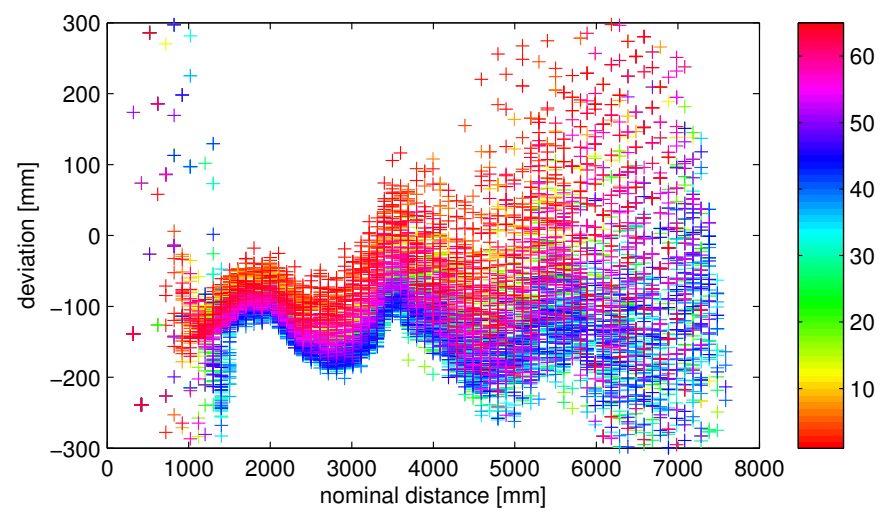

Fig. 7. Deviation as a function of nominal distance: plot of measurements from 60 th row of a range image.

With the proposed calibration method, the accuracy of the distance measurements is improved and the variance as well as the curvature effect of the data is reduced. In Fig. 8, a comparison between a range image before and after calibration is presented.

\subsection{Bad pixel removal}

As mentioned in Section 2, the employed camera is for indoor use only, since background light suppression is not implemented in this PMD camera type. Thus sunlight reflected on a white wall would lead to wrong distance measurements.

Furthermore, measuring the range to highly reflective material like glass, metal or glossy plastic is problematic. As May et al. [2006] reported, light emitting sources lead to inaccuracies and false measurements, due to the infrared components in the light spectrum. However, it is possible to filter measurements by using amplitude thresholds (bad pixel removal), e.g. amplitude mean or amplitude median.

For dynamic scenes (for a moving camera or changing environments), motion noise occurs in the stream of range

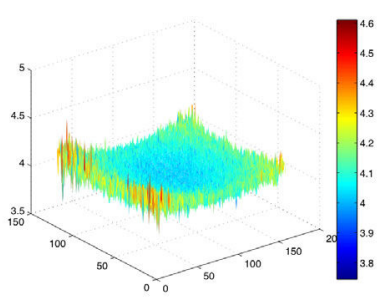

(a)

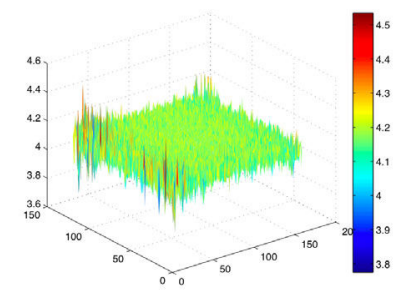

(c)

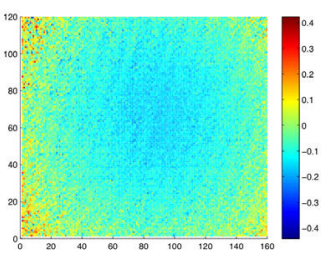

(b)

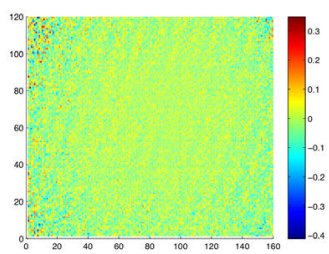

(d)
Fig. 8. Calibration of a range image. All values are in meters. Nominal distance to target is $4.186 \mathrm{~m}$. (a) Measurement with mean distance 4.1093 (b) Deviation from nominal value with norm 12.6863 and standard deviation 0.069 (c) Range image after calibration. distance mean 4.1762 (d) Deviation from nominal value with norm 1.9345 and standard deviation 0.0446 .

images from the camera (Fig. 9). This noise appears especially at edges of objects, where it is then difficult to determine the distance.

In an approach analyzed by Lottner et al. [2007] movement artifacts are detected with an additional video camera and gradient edge detection methods.

From PMD data only, motion noise can be removed to some extend directly by using the fact that problematic edge pixels often own a high intensity value, while having a low amplitude. Hereby, it is possible to detect and remove these wrong edge pixels, by imposing a threshold for intensity and amplitude values (Fig. 9 (c) and (e)):

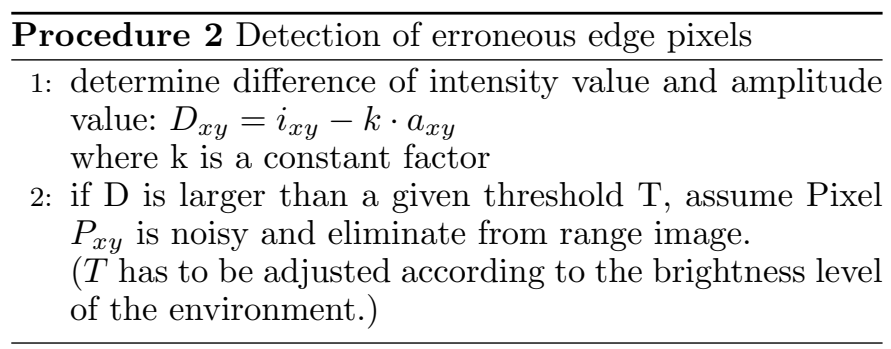

\section{APPLICATIONS}

These measurement results and the proposed adaptation and calibration methods support future use of the range camera for mobile robotics. Potential application areas are navigation support for teleoperators or for autonomous movements, e.g. for map building or obstacle avoidance.

\subsection{Live navigation}

User tests emphasize, that live navigation with PMD range images requires a high cognitive effort from the user. The 


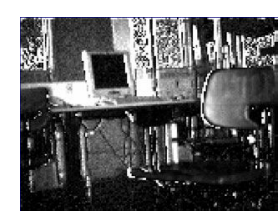

(a)

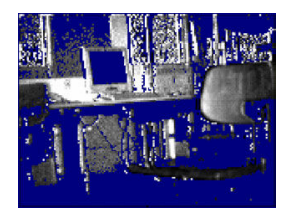

(b)

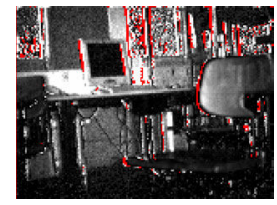

(c)

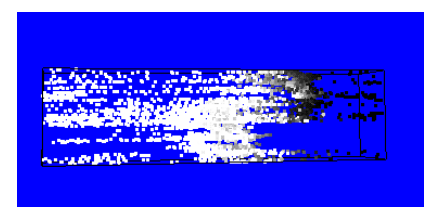

(d)

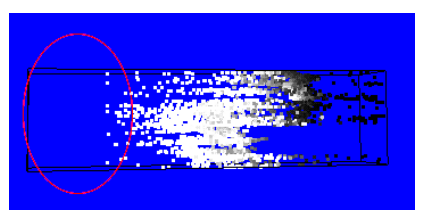

(e)
Fig. 9. Example for filtering: (a) unfiltered image, (b) amplitude threshold is mean amplitude, (c) problematic edge pixels (red) are removed, ( $\mathrm{d}$ and e) distance measurement pixels are from top projected in $2 \mathrm{D}$ plane; camera is placed on the right. In (e) one can recognize that eliminated pixels lie in the back, highlighted by red ellipse.

available field of view is limited and motion noise further complicates perception. Moreover, it is hard to find a descent compromise between update rate and adaptation of integration time, which influences image quality. At the current state, navigation with live distance data from a PMD camera only is not recommended.

\subsection{D panoramas}

A larger field-of-view supports a teleoperator in maintaining situation awareness during the navigation task. An extension of field-of-view can be achieved by rotating the sensor and merging multiple images into one. The sensor can either be moved by a pan tilt unit or by the robot itself. Here, the Iterative Closest Point scan matching algorithm (ICP) was implemented to retrieve $360^{\circ}$ 3D-Panoramas. Furthermore, 3D-Panoramas can be seen as local maps, that describe features to be added to topological map nodes.

\subsection{Map building}

In addition, 3D Panoramas can be used to build global metric 3D maps (Fig. 10). For this purpose, a MonteCarlo localization based on a 2D laser scan was used. Moreover, improved teleoperation and object recognition performance become obvious in user navigation tests. A major advantage of the $3 \mathrm{D}$ map is that the operator can select arbitrary perspectives. Fig. 10 (right side) presents three different perspectives, which can be chosen according to demand. Fig. 11 displays as alternative representation to the point cloud of Fig. 10 an octree representation, which can be advantageous for navigation tasks.

From scan registration, the transform found by the algorithm can be seen as an estimate of the robot's movements. These tracking data provide inputs for the robot's navigation system. In this case real-time matching of scan data needs to be done.

\subsection{Obstacle avoidance}

The potential for obstacle avoidance was tested with the presented adaptation method for integration time. The robot's camera is oriented toward the floor to recognize positive and negative obstacles from the range images. Currently, the data acquisition frequency of the PMD cameras is below the one of typical range sensors in robotics, e.g. ultrasonic sensors. This restricts the robot's maximum safe velocity. The strength of this PMD-camera approach corresponds to the detection of negative obstacles and free paths where other range measurement sensors would detect a blocked path due to their two-dimensional nature.

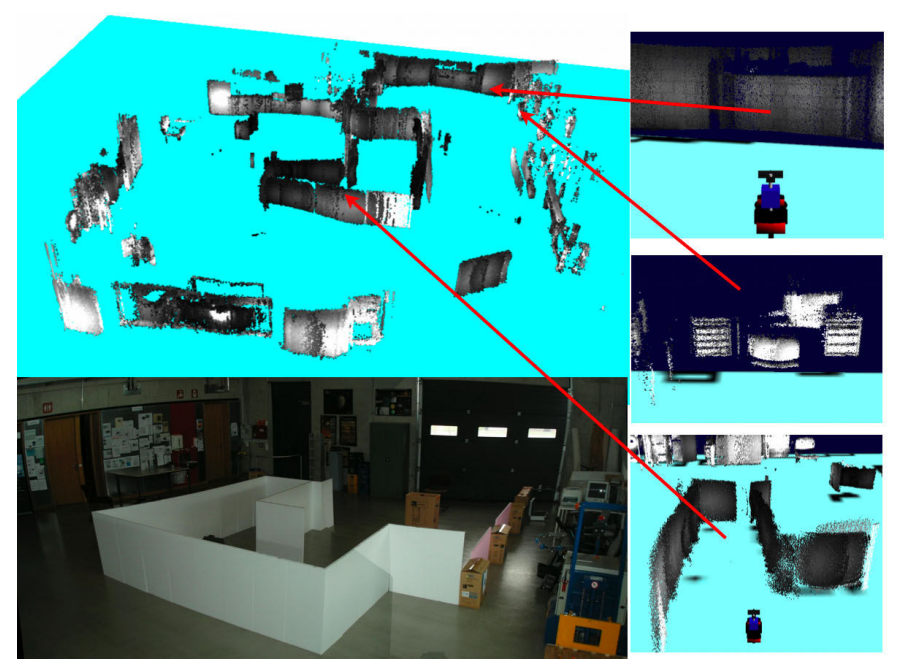

Fig. 10. Picture and global 3D map of test environment. On the right side three different viewpoints are shown (exocentric close, egocentric, exocentric far).

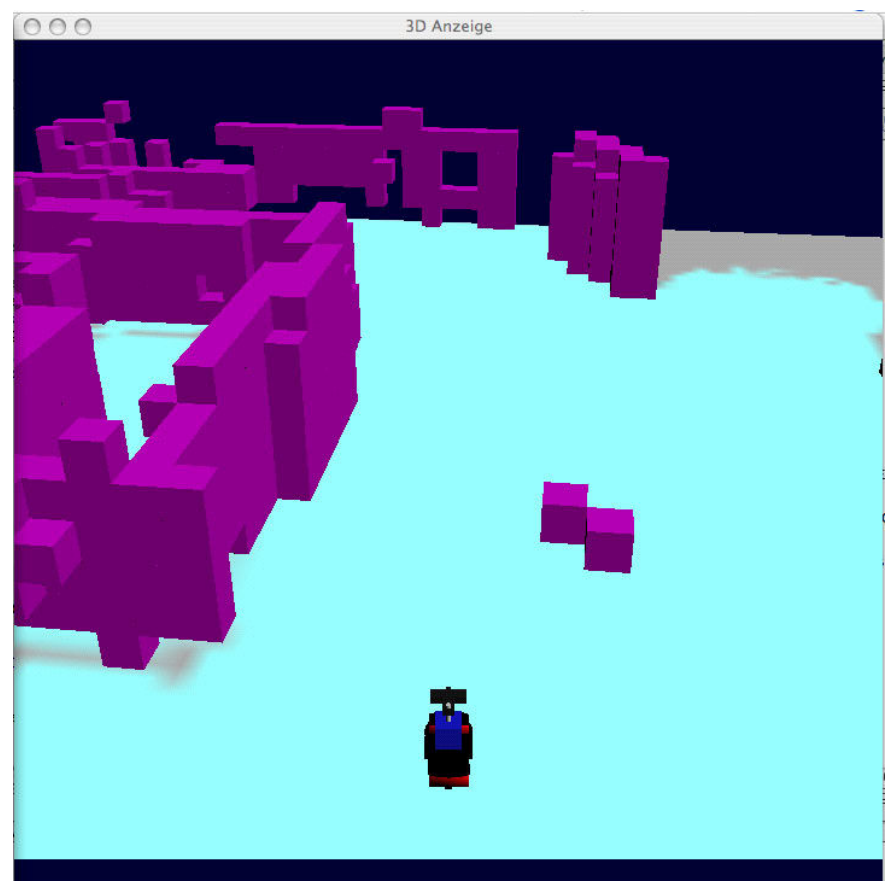

Fig. 11. Representation of range measurement as octree. 


\section{CONCLUSIONS}

PMD-cameras offer interesting potential to capture 3Dimages for mobile vehicle applications. Specific parameter adaptation schemes are proposed in order to improve image quality. This contribution addresses in particular adaptation of integration time, calibration of data, as well as filtering schemes for bad pixels. These methods provided significant improvements of the measurement quality, analyzed in more detail for applications in the field of mobile robots, like map building, navigation support, or obstacle detection.

The tests for using the camera in mobile robotics applications were presented and show promising results for further research. Future work will include evaluating, how sensor types like lasers, or ultrasonic sensors can be combined with PMD camera. Sensor data fusion methods might enable combination of the strengths of these different sensor types for robust environment characterization solutions.

\section{REFERENCES}

Jeff Craighead, Brian Day, and Robin Murphy. Evaluation of canesta's range sensor technology for urban search and rescue and robot navigation. Proceedings of the 2006 International Workshop for Safety, Security and Rescue Robotics (SSRR 2006), August 2006.

F. Driewer, H. Baier, and K. Schilling. Robot/human rescue teams: A user requirement analysis. Advanced Robotics, 19(8):819-838, 2005.

Huan Du, Thierry Oggier, Felix Lustenberger, and Edoardo Charbon. A Virtual Keyboard Based on True3D Optical Ranging. Proceedings of the British Machine Vision Conference, 1:220 - 229, 2005.

H.R. Everett. Sensors for mobile robots. AK Peters, 1995.

T. Hong, R. Bostelman, and R. Madhavan. Obstacle Detection using a TOF Range Camera for Indoor AGV Navigation, PerMIS 2004, Gaithersburg. MD, June, 2004.

Timo Kahlmann, F. Remondino, and H. Ingensand. Calibration for increased accuracy of the range imaging camera swissranger ${ }^{\mathrm{TM}}$. ISPRS Commission V Symposium 'Image Engineering and Vision Metrology', 2006.

Uwe Kiencke and Lars Nielsen. Automotive Control Systems. Springer Verlag, 2000.

Klaus-Dieter Kuhnert and Martin Stommel. Fusion of stereo-camera and pmd-camera data for real-time suited precise 3d environment reconstruction. In International Conference Spatial Cognition, Bremen, 2006.

M. Lindner and A. Kolb. Lateral and depth calibration of pmd-distance sensors. SPIE: Intelligent Robots and Computer Vision XXV, 2:524-533, 2006.

M. Lindner, A. Kolb, and K. Hartmann. Data-fusion of pmd-based distance-information and high-resolution rgb-images. In International Symposium on Signals, Circuits and Systems (ISSCS), 2007.

O. Lottner, A. Sluiter, Hartmann K., and W. Weihs. Movement artefacts in range images of time-of-flight cameras. In International Symposium on Signals, Circuits 86 Systems (ISSCS), 2007.

Stefan May, Björn Werner, Hartmut Surmann, and Kai Pervölz. 3d time-of-flight cameras for mobile robotics. IEEE/RSJ International Conference on Intelligent Robots and Systems, 2006, 2006.
Tobias Möller, Holger Kraft, Jochen Frey, Martin Albrecht, and Robert Lange. Robust 3d measurement with pmd sensors. In Proceedings of the First Range Imaging Research Day at ETH Zurich, 2005.

Andreas Nüchter, Kai Lingemann, Joachim Hertzberg, and Hartmut Surmann. 6D SLAM with approximate data association. Proceedings of the 12th International Conference on Advanced Robotics (ICAR '05), pages 242-249, July 2005.

Andreas Nüchter, Kai Lingemann, and Joachim Hertzberg. Kurt3d - a mobile robot for 3d mapping of environments. ELROB Technical Paper, Hammelburg, Germany, May 2006.

Thierry Oggier, Bernhard Büttgen, and Felix Lustenberger. Swissanger sr3000 and first experiences based on miniaturized 3d-tof cameras. In Proceedings of the First Range Imaging Research Day at ETH Zurich, 2005.

T.D.A. Prasad, K. Hartmann, W. Wolfgang, S.E. Ghobadi, and A. Sluiter. First steps in enhancing 3d vision technique using $2 \mathrm{~d} / 3 \mathrm{~d}$ sensors. In Computer Vision Winter Workshop 2006, pages 82-86, 2006.

Alexander Prusak, Hubert Roth, and Rudolf Schwarte. Application of 3d-pmd video cameras for tasks in the autonomous mobile robotics. 16th IFAC World Congress Prague, Czech Republic, from July 4 to July 8, 2005.

Niramon Ruangpayoongsak. Development of autonomous features and indoor localization techniques for car-like mobile robots. PhD thesis, Universität Siegen, 2006.

K. Schilling. Control of planetary rovers. Special Issue of Control Engineering Practice, 5:823ff, 1997.

K. Schilling and F. Driewer. Remote control of mobile robots for emergencies. In 16th IFAC World Congress, Prague, Czech Republic, 2005.

K. Schilling, M. Mellado, P. Lopez, and J. Remm. Free navigating autonomous transport robots for flexible industrial production. In N. Mårtensson, R. Mackay, and S. Björgvinsson, editors, Changing the Ways We Work, pages $674-684$. IOS Press, 1998.

Raymond Sheh, Nawid Jamali, M. Waleed Kadous, and Claude Sammut. A low-cost, compact, lightweight 3d range sensor. In Proceedings Australian Conference on Robotics and Automation, Auckland, New Zealand, 2006.

J. Weingarten and R. Siegwart. Ekf-based 3d slam for structured environment reconstruction. In Proceedings of IROS 2005, 2005.

J. Weingarten, G. Gruener, and R. Siegwart. A state-ofthe-art $3 \mathrm{~d}$ sensor for robot navigation. In Proceedings of IROS 2004, 2004.

Oliver Wulf and Bernardo Wagner. Fast 3d scanning methods for laser measurement systems. International Conference on Control Systems and Computer Science (CSCS14), July 2003.

Z. Xu, R. Schwarte, H. Heinol, B. Buxbaum, and T. Ringbeck. Smart pixel - photonic mixer device (pmd). Proc. M2VIP' 98 - International Conference on Mechatronics and Machine Vision in Practice, pages 259 - 264, 1998. 